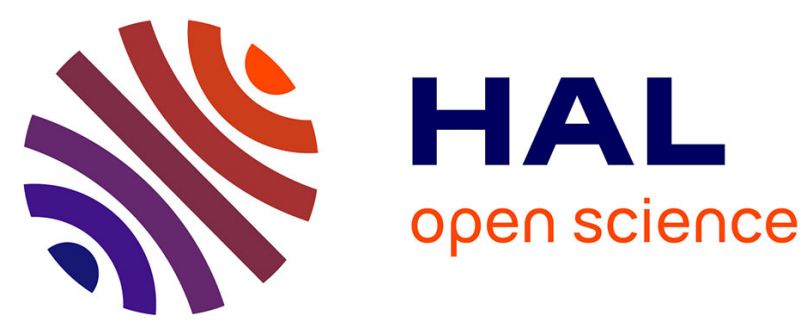

\title{
Stability analysis for bacterial linear metabolic pathways with monotone control system theory
}

Nacim Meslem, Vincent Fromion, Anne Goelzer, Laurent Tournier

\section{To cite this version:}

Nacim Meslem, Vincent Fromion, Anne Goelzer, Laurent Tournier. Stability analysis for bacterial linear metabolic pathways with monotone control system theory. 7. International Conference on Informatics in Control, Automation and Robotics, Jun 2010, Funchal, Madeira, Portugal. hal-02753049

\author{
HAL Id: hal-02753049 \\ https://hal.inrae.fr/hal-02753049
}

Submitted on 3 Jun 2020

HAL is a multi-disciplinary open access archive for the deposit and dissemination of scientific research documents, whether they are published or not. The documents may come from teaching and research institutions in France or abroad, or from public or private research centers.
L'archive ouverte pluridisciplinaire HAL, est destinée au dépôt et à la diffusion de documents scientifiques de niveau recherche, publiés ou non, émanant des établissements d'enseignement et de recherche français ou étrangers, des laboratoires publics ou privés. 


\title{
STABILITY ANALYSIS FOR BACTERIAL LINEAR METABOLIC PATHWAYS WITH MONOTONE CONTROL SYSTEM THEORY
}

\author{
Nacim Meslem, Vincent Fromion, Anne Goelzer and Laurent Tournier \\ INRA, Mathematics, Informatics and Genome Laboratory \\ Jouy-en-Josas, Domaine de Vilvert 78352 Jouy-en-Josas Cedex, France \\ \{nmeslem,vfromion, agoelzer, ltournier\}@jouy.inra.fr
}

Keywords: Monotone control systems, Negative feedback theorem, Linear bacterial metabolic pathways.

\begin{abstract}
In this work we give technical conditions which guarantee the global attractivity of bacterial linear metabolic pathways (reversible and irreversible structures) where both genetic and enzymatic controls involve the end product through metabolic effectors. To reach this goal, we use the negative feedback theorem of the monotone control systems theory, and we represent all conditions needed to apply the negative feedback theorem to the bacterial linear metabolic pathways in convenient deduced forms.
\end{abstract}

\section{INTRODUCTION}

The bacterial metabolic machinery and its regulation make up a complex system involving many cellular components such as metabolites and enzymes. In this paper, we focus on the dynamical behavior of the control structures used in a large number of bacterial biosynthesis pathways where both the genetic and enzymatic controls involve the last product as metabolite effector (Goelzer et al., 2008). Stability analysis of these biological structures is recognized as an issue of great importance in order to deduce key biological properties of the bacterial metabolic pathways. In the literature, many studies focused on the analysis of the metabolic and genetic networks separately. For instance, using the stability results about cyclic dynamical systems (Tyson and Othmer, 1978), (Sanchez, 2009), (Arcak and Sontag, 2006), one can state nice stability conditions of the irreversible linear metabolic pathways with allosteric regulation. One can also use the stability results about tridiagonal systems (Angeli and Sontag, 2008), (Wang et al., 2008) to analyze the stability of the reversible metabolic pathways. However, few works have considered structures with both genetic and allosteric regulation. Thus, in this paper we investigate stability of the common structures shared by many bacteria cells and yeasts. These structures are called end product structures, because both genetic and enzymatic controls involve the end product of the pathway (Grundy et al., 2003), (Gollnick et al., 2005), (Goelzer et al., 2008).

We will use the monotone control system theory developed in (Angeli and Sontag, 2003) to deal with stability issue of biological systems. In particular, the negative feedback theorem has been applied to a model of Mitogen-Activated Protein Kinase (MAPK) cascades in (Angeli and Sontag, 2003), and more recently to Goldbeter's circadian model (Angeli and Sontag, 2008). The main contribution of this work consists in providing technical conditions to check all the required assumptions to apply the negative feedback theorem to end product structures (under irreversible and reversible forms).

This paper is structured as follows. Section 2 presents the mathematical models for the linear reversible and irreversible bacterial metabolic pathways and states the main results of this paper which consist in propositions 1 and 2 . Section 3 recalls some definitions and properties of monotone control systems theory and introduces the negative feedback theorem. Section 4 addresses the stability analysis of the dynamical models introduced in section 2 and proves the two propositions.

\section{LINEAR METABOLIC PATHWAYS}

Consider a linear pathway with $n$ metabolites involved in enzymatic reactions, an input flux $v_{1}$ and an output flux $v_{n}$ as depicted in Figure 1. Each $X_{i}$ and $\mathbb{E}_{i}$ correspond to a metabolite and an enzyme respectively. We assume that the pool $X_{1}$ of the first 


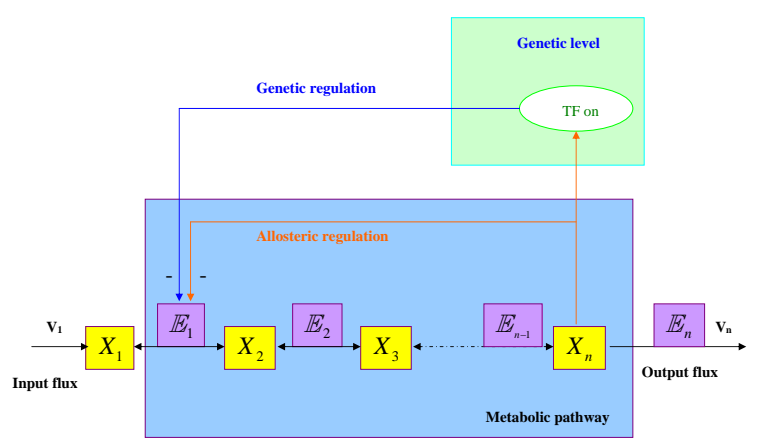

Figure 1: End product control linear structure.

metabolite is maintained by the input flux $v_{1}$ which corresponds to a supply flux. Hence its concentration $\bar{x}_{1}$ is strictly positive constant. The output of the pathway is the flux $v_{n}$ which corresponds to the bacterium requirement for the metabolite $X_{n}$. Hereafter, for each $i \in\{2, \ldots, n\}$ we denote by $x_{i}$ the nonnegative concentration of the metabolite $X_{i}$, and by $E_{i}$ the assumed constant positive concentration of the enzyme $\mathbb{E}_{i}$. The three phenomena, enzymatic reactions, allosteric regulation and genetic regulation (with respect to $E_{1}$ ), presented in Figure 1 can be described by a set of interconnected nonlinear differential equations. In the sequel, we analyze global stability of two types of the interconnected differential equations, namely the reversible and irreversible metabolic pathways.

\subsection{Reversible Pathways}

The common end product structure of linear reversible metabolic pathways is described by the following dynamical system:

$$
\left\{\begin{array}{ccc}
\dot{x}_{2} & = & E_{1} f_{1}\left(\bar{x}_{1}, x_{2}, x_{n}\right)-E_{2} f_{2}\left(x_{2}, x_{3}\right) \\
\dot{x}_{3} & = & E_{2} f_{2}\left(x_{2}, x_{3}\right)-E_{3} f_{3}\left(x_{3}, x_{4}\right) \\
\vdots & \vdots & \vdots \\
\dot{x}_{n} & = & E_{n-1} f_{n-1}\left(x_{n-1}, x_{n}\right)-E_{n} f_{n}\left(x_{n}\right) \\
\dot{E}_{1} & = & g\left(x_{n}\right)-\mu E_{1}
\end{array}\right.
$$

where the Lipschitz functions $f_{i}$ denote the reaction rates of the enzymes $\mathbb{E}_{i}$. Note that, in the reversible structures all reaction rates depend on the product and substrate concentrations and have the following properties:

- For the first enzyme: we assume that the metabolite $X_{n}$ modulates the activity of the enzyme $\mathbb{E}_{1}$ through, for example, an allosteric effect. The function $f_{1}\left(x_{1}, x_{2}, x_{n}\right)$ is increasing in its first argument and decreasing with respect to its second and third arguments, and we have for any $x_{1}>0$, $x_{2} \geq 0$ and $x_{n} \geq 0, f_{1}\left(x_{1}, x_{2}, x_{n}\right)>0$ and for any $x_{n} \geq 0, f_{1}\left(0,0, x_{n}\right)=0$. In addition, there exists
$M_{1}>0$ such that for any $x_{1}>0, x_{2} \geq 0$ and $x_{n} \geq 0$, $f_{1}\left(x_{1}, x_{2}, x_{n}\right) \in\left[0, M_{1}\right)$. We also assume that for any $x_{1}>0$ and $x_{n} \geq 0$ there exists $x_{2}^{*}>0$ such that $f_{1}\left(x_{1}, x_{2}^{*}, x_{n}\right)=0$. Finally, for any $x_{1}>0$ and $x_{2}>0$ we have,

$$
\lim _{x_{n} \rightarrow+\infty} f_{1}\left(x_{1}, x_{2}, x_{n}\right)=0 .
$$

- For the intermediate enzymes: $f_{i}, i \in\{2, \ldots, n-$ $1\}$, is increasing in $x_{i}$ and decreasing in $x_{i+1}$. For any $x_{i}>0, f_{i}\left(x_{i}, 0\right)>0$, and for any $x_{i+1}>$ $0, f_{i}\left(0, x_{i+1}\right)<0$ and $f_{i}(0,0)=0$. Moreover, there exists $M_{i}>0$ and $M_{i}^{\prime} \geq 0$ such that for any $x_{i}>0$ and $x_{i+1} \geq 0, f_{i}\left(x_{i}, x_{i+1}\right) \in\left(-M_{i}^{\prime}, M_{i}\right)$. Finally, we assume that for any $x_{i}>0$ there exists $x_{i+1}^{*}>0$ such that $f_{i}\left(x_{i}, x_{i+1}^{*}\right)=0$.

- For the final enzyme: $\mathbb{E}_{n}$ describes the properties of the remainder part of the metabolic network and summarizes the relation between the flux supplied by the pathway and the final concentration. The properties of $f_{n}$ mainly depends on the properties of the next modules, and generally $f_{n}$ is a strictly increasing, positive and bounded function in $x_{n}$ such that

$$
f_{n}(0)=0, \lim _{x_{n} \rightarrow+\infty} f_{n}\left(x_{n}\right)=M_{n} .
$$

The dynamics of the enzyme concentrations during the exponential growth phase are mostly the result of two phenomena: (i) the de novo production (ii) the $d i$ lution effect caused by the increase of the cell volume. For this, in the last equation of (1), we have considered that the control of the concentration of the first enzyme is regulated by the concentration of the final metabolite $x_{n}$, where $\mu$ is the growth rate of the bacterium assumed to be in the exponential growth phase. The term $g\left(x_{n}\right)$ corresponds to the instantaneous production of the enzyme $E_{1}$ modulated by a metabolite (implicitly through a transcription factor). The continuous function $g($.$) is positive strictly decreasing in$ the end product $x_{n}$ with $g(0)=g_{\max }, g_{\max }>0$ and

$$
\lim _{x \rightarrow+\infty} g(x)=0 \text {. }
$$

After the detailed description of the dynamical model of the linear reversible metabolic pathway, we state below the main results of this paper about its global attractivity.

Stability Results. Let us start by setting three hypotheses and then we introduce our first proposition.

- Hypothesis $\mathcal{H}_{1}$ : The $n-1 \times n-1$ Tridiagonal matrix, 


$$
\mathbf{Q}=\left[\begin{array}{cccccc}
q_{2,2} & q_{2,3} & 0 & \ldots & \ldots & 0 \\
q_{3,2} & q_{3,3} & q_{3,4} & \ddots & & \vdots \\
0 & \ddots & \ddots & \ddots & \ddots & \vdots \\
\vdots & \ddots & \ddots & \ddots & \ddots & 0 \\
\vdots & & \ddots & q_{n-1, n-2} & q_{n-1, n-1} & q_{n-1, n} \\
\vdots & \ldots & \ldots & 0 & q_{n, n-1} & q_{n, n}
\end{array}\right]
$$

where $\forall i, j \in\{2, \ldots, n\}$

$$
q_{i, j}=\sup \left(\frac{\partial\left(E_{i-1} f_{i-1}(.)-E_{i} f_{i}(.)\right)}{\partial x_{j}}\right),
$$

is Hurwitz.

- Hypothesis $\mathcal{H}_{2}$ : The inequality $E_{n-1} M_{n-1} \leq$ $E_{n} M_{n}$ is verified.

- Hypothesis $\mathcal{H}_{3}$ : The graph of the scalar function

$$
T(u)=g \circ k_{y}(u)
$$

and that of its reciprocal function $T^{-1}(u)$ have a unique intersection point on the open interval $u \in$ $\left(0, g_{\max }\right)$.

The scalar function $k_{y}($.$) is the static input-output$ characteristic associated to the monotone part of (1) (resp. (2)), see Definition 2 in subsection 3.1.

Proposition 1. If $\mathcal{H}_{1}, \mathcal{H}_{2}$ and $\mathcal{H}_{3}$ are satisfied, then for any $\bar{x}_{1}$ and $E_{n}$, the reversible end product structure (1) has globally attractive equilibrium.

\subsection{Irreversible Pathways}

The main difference between the irreversible and the reversible metabolic pathways is in the reaction rates $f_{i}$ for the first and intermediate enzymes. Indeed, here we assume that the reaction rates depend only on the substrate concentration and have the following properties:

- For the first enzyme. We assume that the function $f_{1}$ is increasing in its first argument and decreasing in its second argument and for any $x_{1}>0$,

$$
\lim _{x_{n} \rightarrow+\infty} f_{1}\left(x_{1}, x_{n}\right)=0 .
$$

In addition, we have for any $x_{n} \geq 0, f_{1}\left(0, x_{n}\right)=0$ and there exists $M_{1}>0$ such that for any $x_{1}>0$ and $x_{n} \geq 0, f_{1}\left(x_{1}, x_{n}\right) \in\left[0, M_{1}\right)$.

- For the intermediate enzymes: $f_{i} i \in\{2, \ldots, n-1\}$ is strictly increasing in $x_{i}$ and $f_{i}(0)=0$. Moreover, there exists $M_{i}>0$ such that

$$
\lim _{x_{i} \rightarrow+\infty} f_{i}\left(x_{i}\right)=M_{i} \text {. }
$$

Then, the end product structure of the linear irreversible metabolic pathways is described by the following dynamical system

$$
\left\{\begin{array}{ccc}
\dot{x}_{2} & = & E_{1} f_{1}\left(\bar{x}_{1}, x_{n}\right)-E_{2} f_{2}\left(x_{2}\right) \\
\dot{x}_{3} & = & E_{2} f_{2}\left(x_{2}\right)-E_{3} f_{3}\left(x_{3}\right) \\
\vdots & \vdots & \vdots \\
\dot{x}_{n} & = & E_{n-1} f_{n-1}\left(x_{n-1}\right)-E_{n} f_{n}\left(x_{n}\right) \\
\dot{E}_{1} & = & g\left(x_{n}\right)-\mu E_{1} .
\end{array}\right.
$$

Stability Results. Now, we state the contribution of this paper concerning the global attractivity of the irreversible metabolic pathway (2).

- Hypothesis $\mathcal{H}_{4}$ : for each $i \in\{2, \ldots, n\}$ the inequality is verified $\bar{E}_{1} M_{1} \leq E_{i} M_{i}$, where $\bar{E}_{1}$ is the upper bound of all solutions $E_{1}(t)$.

Proposition 2. The irreversible end product structure (2) has globally attractive equilibrium for any $\bar{x}_{1}$ and $E_{n}$ if hypotheses $\mathcal{H}_{3}$ and $\mathcal{H}_{4}$ are satisfied.

To prove Proposition 1 and Proposition 2, we will use the monotone control system theory, in particular the negative feedback theorem. Thus, we present briefly this theory in the next section and then we give the proofs in section 4 .

\section{MONOTONE CONTROL SYSTEMS}

Monotone control systems theory (Angeli and Sontag, 2003) is an extension of the autonomous monotone system theory (Smith, 1995). Briefly, monotone control system is a dynamical system on an ordered metric space which has the property that ordered initial states and ordered inputs generate ordered state trajectories and ordered outputs. In other words, a controlled dynamical system (3),

$$
\left\{\begin{array}{rl}
\dot{\mathbf{x}}(t) & =\mathbf{f}(\mathbf{x}(t), \mathbf{u}(t)) \\
\mathbf{y}(t) & =\mathbf{h}(\mathbf{x})
\end{array}, \mathbf{x}\left(t_{0}\right)=c s t\right.
$$

where $\mathbf{x}(t) \in \mathbb{X} \subseteq \mathbb{R}^{n}$ and $\mathbf{u}(t) \in \mathbb{U} \subseteq \mathbb{R}^{m}$, is said monotone if the following implication holds: $\forall\left(\mathbf{x}_{1}\left(t_{0}\right), \mathbf{x}_{2}\left(t_{0}\right)\right) \in \mathbb{X}^{2}$ and $\forall\left(\mathbf{u}_{1}(t), \mathbf{u}_{2}(t)\right) \in \mathbb{U}^{2}$,

$$
\begin{aligned}
& \mathbf{x}_{1}\left(t_{0}\right) \preceq \mathbf{x}_{2}\left(t_{0}\right), \mathbf{u}_{1}(t) \preceq \mathbf{u}_{2}(t) \Rightarrow \\
& \mathbf{x}_{1}\left(t, \mathbf{x}_{1}\left(t_{0}\right), \mathbf{u}_{1}(t)\right) \preceq \mathbf{x}_{2}\left(t, \mathbf{x}_{2}\left(t_{0}\right), \mathbf{u}_{2}(t)\right) \forall t \geqslant t_{0}
\end{aligned}
$$

where $\mathbf{x}\left(t, \mathbf{x}\left(t_{0}\right), \mathbf{u}(t)\right)$ represent the state trajectory generated by (3) with $\mathbf{x}\left(t_{0}\right)$ as initial state and $\mathbf{u}(t)$ as input. The dimensions of the vectors $\mathbf{x}, \mathbf{u}$ and $\mathbf{y}$ are respectively $n, m$ and $p$.

Here, we consider that $\preceq$ is the classical lower or equal comparison operator $\leq$, applied component by 
component. Systems that are monotone with respect to this order are called cooperative systems, as all state variables have a positive influence on one other and the inputs act positively on state variables.

Proposition 3. The dynamical system (3) is cooperative if and only if the following properties hold:

$$
\begin{array}{ll}
\frac{\partial f_{i}}{\partial x_{j}}(\mathbf{x}, \mathbf{u}) \geq 0 & \forall \mathbf{x} \in \mathbb{X}, \forall \mathbf{u} \in \mathbb{U}, \forall i \neq j \\
\frac{\partial f_{i}}{\partial u_{j}}(\mathbf{x}, \mathbf{u}) \geq 0 & \forall \mathbf{x} \in \mathbb{X}, \forall \mathbf{u} \in \mathbb{U}, \forall i, j \\
\frac{\partial h_{i}}{\partial x_{j}}(\mathbf{x}) \geq 0 & \forall \mathbf{x} \in \mathbb{X}, \forall i, j
\end{array}
$$

Proof. See (Angeli and Sontag, 2003; Angeli and Sontag, 2004).

After this brief recall about monotone control systems, we now introduce in the next the negative feedback theorem which states stability conditions for monotone control systems with negative feedback.

\subsection{Stability Analysis with Monotone Control System}

Recently, the negative feedback theorem of the monotone control system theory is used to analyze stability of several biological systems. Indeed, this theorem allows, under some conditions, to obtain the globally attractive stable steady state of non-monotone dynamical systems. Here we give some definitions and assumptions needed to state the negative feedback theorem.

Definition 1 (Angeli and Sontag, 2003). We say that the SISO dynamical system $(3)(m=p=1)$ admits an input to state static characteristic $\mathbf{k}_{x}():. \mathbb{U} \rightarrow \mathbb{X}$ if, for each constant input $u \in \mathbb{U}$, there exists a unique globally asymptotically stable equilibrium noted $\mathbf{k}_{x}(u)$.

Definition 2 (Angeli and Sontag, 2003). SISO system with an input-state characteristic and with a continuous output map $y=h(\mathbf{x})$ has an input to output characteristic defined as the composite function $k_{y}(u)=\left(h \circ \mathbf{k}_{x}\right)(u)$.

Note that, if the system (3) (with $m=p=1$ ) is cooperative and admits a static input-state characteristic $\mathbf{k}_{x}$ and static input-output characteristic $k_{y}$, then $\mathbf{k}_{x}$ and $k_{y}$ must be increasing with respect to $u$, viz.

$$
\forall\left(u_{1}, u_{2}\right) \in \mathbb{U}^{2}, u_{1} \geq u_{2} \Leftrightarrow \begin{aligned}
& \mathbf{k}_{x}\left(u_{1}\right) \geq \mathbf{k}_{x}\left(u_{2}\right), \\
& \\
& k_{y}\left(u_{1}\right) \geq k_{y}\left(u_{2}\right) .
\end{aligned}
$$

Assumptions. Consider the non-monotone autonomous system given by (6)

$$
\dot{\mathbf{x}}(t)=\mathbf{F}(\mathbf{x}),
$$

and let us state the following assumptions,
- $\mathcal{H}_{5}$ : Any state trajectory generated by system (6) is bounded.

- $\mathcal{H}_{6}$ : System (6) is decomposable into an open loop SISO monotone control system (7)

$$
\left\{\begin{aligned}
\dot{\mathbf{x}}(t) & =\mathbf{f}(\mathbf{x}, u) \\
y(t) & =h(\mathbf{x})
\end{aligned}\right.
$$

closed by a monotone decreasing feedback law $f_{b}: y \longrightarrow u$ as depicted in Figure 2 .

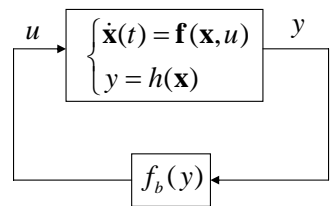

Figure 2: System (6) in closed loop configuration.

- $\mathcal{H}_{7}$ : Open loop system (7) admits a well-defined static input-output characteristic $k_{y}($.$) .$

Then, we can introduce the negative feedback theorem.

Theorem 1. Let (8) be a discrete scalar dynamical system associated to the continuous non-monotone system (6)

$$
u_{j+1}=\left(f_{b} \circ k_{y}\right)\left(u_{j}\right) .
$$

If this iteration has a globally attractive fixed point $u^{*}$ on an open interval $\mathcal{U}_{x}$, then the autonomous system (6), provided that the assumptions $\mathcal{H}_{5}, \mathcal{H}_{6}$ and $\mathcal{H}_{7}$ are satisfied, has a globally attracting steady state $\mathbf{x}^{*}=$ $\mathbf{k}_{x}\left(u^{*}\right)$.

Proof. See (Angeli and Sontag, 2003).

Hereafter, we give proofs of our main results stated in subsections 2.1 and 2.2.

\section{PROOF OF THE MAIN RESULTS}

In this section, we prove that propositions 1 and 2 are consequences of Theorem 1 . We start with the irreversible metabolic pathways, for which the static input-state characteristic of its monotone part is easier to establish. Then we will focus on the reversible pathways.

\subsection{Irreversible Structure}

In this subsection we will show that the technical Proposition 2 is a consequence of Theorem 1. 
Checking Assumption $\mathcal{H}_{5}$. First of all, let us prove the boundedness of the controlled enzyme $E_{1}$ which is governed by the following differential equation

$$
\dot{E}_{1}=g\left(x_{n}\right)-\mu E_{1} \text {. }
$$

By definition we know that $g($.$) is bounded, viz.$ $\forall x_{n}, g\left(x_{n}\right) \in\left(0, g_{\max }\right]$. Then, for any $x_{n}$ the solution $E_{1}(t)$ of (9) is framed by

$$
\check{E}_{1}(t) \leq E_{1}(t) \leq \hat{E}_{1}(t),
$$

where $\breve{E}_{1}(t)$ and $\hat{E}_{1}(t)$ are respectively the solutions of the following stable linear differential equations

$$
\dot{\check{E}}_{1}=-\mu \check{E}_{1} \text { and } \dot{\hat{E}}_{1}=g_{\max }-\mu \hat{E}_{1} \text {. }
$$

Thus, there exists

$$
\bar{E}_{1}>0 \mid \forall t \geq 0, E_{1}(t) \leq \bar{E}_{1} .
$$

Now, consider the first differential equation of (2)

$$
\begin{aligned}
\dot{x}_{2} & =E_{1} f_{1}\left(\bar{x}_{1}, x_{n}\right)-E_{2} f_{2}\left(x_{2}\right) \\
& \leq E_{1} M_{1}-E_{2} f_{2}\left(x_{2}\right) .
\end{aligned}
$$

We know that $f_{2}($.$) is positive increasing and$ bounded. Then if

$$
\bar{E}_{1} M_{1} \leq E_{2} M_{2},
$$

there exists $x_{2}^{\star}$ such that $E_{2} f_{2}\left(x_{2}^{\star}\right)=\bar{E}_{1} M_{1}$, and we obtain

$$
\forall x_{2}>x_{2}^{\star}, \dot{x}_{2} \leq 0,
$$

namely the solution $x_{2}(t)$ decreases towards $x_{2}^{\star}$ and then the metabolite concentration $x_{2}$ is bounded. In addition, for any initial condition $x_{2}\left(t_{0}\right)$ there exists $t^{\star} \geq t_{0}$ such that,

$$
\forall t \geq t^{\star}, E_{2} f_{2}\left(x_{2}(t)\right) \leq E_{2} f_{2}\left(x_{2}^{\star}\right)=\bar{E}_{1} M_{1} .
$$

To proof the boundedness of the remainder metabolite concentrations, we use mathematical induction. Assume that $x_{i}$ is bounded, viz. the following inequality is satisfied

$$
\bar{E}_{1} M_{1} \leq E_{i} M_{i},
$$

and there exists $\left(t^{\star}, x_{i}^{\star}\right)$ such that for all $t \geq t^{\star}$

$$
E_{i} f_{i}\left(x_{i}(t)\right) \leq E_{i} f_{i}\left(x_{i}^{\star}\right)=\cdots=E_{2} f_{2}\left(x_{2}^{\star}\right)=\bar{E}_{1} M_{1} .
$$

Then, for $t \geq t^{\star}$ the dynamics of the next metabolite concentration $x_{i+1}$ is bounded by

$$
\begin{aligned}
\dot{x}_{i+1} & =E_{i} f_{i}\left(x_{i}\right)-E_{i+1} f_{i+1}\left(x_{i+1}\right) \\
& \leq E_{i} f_{i}\left(x_{i}^{\star}\right)-E_{i+1} f_{i+1}\left(x_{i+1}\right) \\
& =E_{1} M_{1}-E_{i+1} f_{i+1}\left(x_{i+1}\right) .
\end{aligned}
$$

Hence we show, with the same way used to prove the boundedness of $x_{2}$, that inequality (12) guarantees the boundedness of the metabolite concentration $x_{i+1}$.

$$
\bar{E}_{1} M_{1} \leq E_{i+1} M_{i+1} \text {. }
$$

Therefore $\mathcal{H}_{4}$ guarantees the boundedness of the all state trajectories generated by (2), namely $\mathcal{H}_{5}$.
Checking Assumption $\mathcal{H}_{6} \cdot$ System (2) is not monotone. However, we can regard it as a cooperative controlled system (13), which has a triangular Jacobian matrix $D F(\mathbf{x})$ with nonnegative off-diagonal entries, closed by a negative feedback (14),

- Open loop (cooperative system)

$$
\left\{\begin{array}{cccc}
\dot{x}_{2} & = & E_{1} f_{1}\left(\bar{x}_{1}, g^{-1}(u)\right)-E_{2} f_{2}\left(x_{2}\right) \\
\dot{x}_{3} & = & E_{2} f_{2}\left(x_{2}\right)-E_{3} f_{3}\left(x_{3}\right) \\
\vdots & \vdots & \vdots & \vdots \\
\dot{x}_{n} & = & E_{n-1} f_{n-1}\left(x_{n-1}\right)-E_{n} f_{n}\left(x_{n}\right) \\
\dot{E}_{1} & = & u-\mu E_{1} \\
y & = & x_{n}
\end{array}\right.
$$

- Negative feedback

$$
u=g(y)
$$

where $g^{-1}($.$) is the reciprocal function of g($.$) and u \in$ $\left(0, g_{\text {max }}\right)$ since $g(.) \in\left(0, g_{\text {max }}\right]$. This verifies $\mathcal{H}_{6}$.

Checking Assumption $\mathcal{H}_{7}$. The static input-state characteristic $\mathbf{k}_{x}(u)$ of (13) is computed at steady states corresponding to constant inputs $u$. Thus, we vanish all the time derivatives of (13) to obtain:

$$
\mathbf{k}_{x}^{T}(u)=\left[f_{2}^{-1}\left(\frac{f_{1}\left(\bar{x}_{1}, g^{-1}(u)\right) u}{E_{2} \mu}\right), \ldots, f_{n}^{-1}\left(\frac{f_{1}\left(\bar{x}_{1}, g^{-1}(u)\right) u}{E_{n} \mu}\right), \frac{u}{\mu}\right]
$$

and for the static input-output characteristic we have:

$$
k_{y}(u)=f_{n}^{-1}\left(\frac{f_{1}\left(\bar{x}_{1}, g^{-1}(u)\right) u}{E_{n} \mu}\right)
$$

Since functions $f_{i}(),. i=2, \ldots, n$ are bounded, the existence of (15) is conditioned by the following in equalities:

$$
\forall i, \forall u \in\left(0, g_{\max }\right), \frac{f_{1}\left(\bar{x}_{1}, g^{-1}(u)\right) u}{E_{i} \mu} \leq M_{i}
$$

which are always true if assumption $\mathcal{H}_{4}$ is verified. Moreover, as system (13) is cooperative, both static characteristics ((15) and (16)) are increasing with respect to $u$.

Now, to prove that for each constant input $u \in$ $\left(0, g_{\text {max }}\right)$ there exists a unique globally asymptotically stable equilibrium point $\mathbf{k}_{x}(u)$ for (13), we consider separately the dynamics of the enzymatic reactions $\left(\dot{x}_{2}, \ldots, \dot{x}_{n}\right)^{T}$ and that of the genetic regulation $\dot{E}_{1}$.

- The growth rate $\mu$ of the bacteria is constantly positive. Then for each constant input $u$ all the solutions generated by the dynamics of the genetic regulation converge asymptotically to $\frac{u}{\mu}$.

- The Jacobian matrix $D F(\mathbf{x})$ of the dynamics of the enzymatic reactions is a lower triangular matrix with nonnegative off-diagonal entries and real 
negative eigenvalues. Then $-D F(\mathbf{x})$ is a $M$ Matrix (Berman and Plemmons, 1994) and there exists a diagonal matrix $\mathbf{P}=\operatorname{diag}\left(p_{1}, \ldots, p_{n}\right)$ with $p_{i}>0$ such that

$$
\exists \varepsilon>0, \forall \mathbf{x}, \quad \mathbf{P} D F(\mathbf{x})+D F(\mathbf{x})^{T} \mathbf{P}<-\varepsilon \mathbf{I}_{n-1} .
$$

Consequently, we can state that the dynamics of the enzymatic reactions have a well defined quadratic Lyapunov function:

$$
V(\mathbf{z})=\mathbf{z}^{T} \mathbf{P} \mathbf{z}
$$

where $\mathbf{z}=\mathbf{x}-\mathbf{x}^{*}, x_{i}^{*}=\left(k_{x}(u)\right)_{i}, i=2, \ldots, n$ and

$$
\begin{aligned}
\dot{V}(\mathbf{z}) & =\mathbf{z}^{T} \mathbf{P}\left[\mathbf{f}(\mathbf{x}, u)-\mathbf{f}\left(\mathbf{x}^{*}, u\right)\right] \\
& =\mathbf{z}^{T} \int_{0}^{1} \mathbf{P} D F(\lambda \mathbf{z}+\mathbf{x}) \mathbf{z} d \lambda \\
& =\frac{1}{2} \mathbf{z}^{T} \int_{0}^{1}\left(\mathbf{P} D F(\lambda \mathbf{z}+\mathbf{x})+D F(\lambda \mathbf{z}+\mathbf{x})^{T} \mathbf{P}\right) d \lambda \mathbf{z} \\
& \leq-\frac{1}{2} \varepsilon\|\mathbf{z}\|^{2}
\end{aligned}
$$

Hence, for each constant input $u \in\left(0, g_{\text {max }}\right)$, any solution of the open loop system (13) converges asymptotically to the unique steady state given by (15). This verifies assumption $\mathcal{H}_{7}$.

Now, to complete the proof that the Proposition 2 is consequence of Theorem 1, we will show that assumption $\mathcal{H}_{3}$ implies the global attractivity of the following scalar discrete dynamical system

$$
u_{j+1}=g\left(f_{n}^{-1}\left(\frac{f_{1}\left(\bar{x}_{1}, g^{-1}\left(u_{j}\right)\right) u_{j}}{E_{n} \mu}\right)\right)
$$

To do so, (i) we prove existence and unicity of a fixed point $u^{*}$ for (19); and (ii) we give convenient condition which guarantee its global attractivity.

Existence and Unicity. To prove this property, it is sufficient to show that the curves of the functions $g^{-1}(u)$ and $k_{y}(u)$ have a unique intersection point over the interval $\left(0, g_{\max }\right)$. Since:

- $k_{y}(u)$ is is monotone increasing with respect to $u$ and for $u=0, k_{y}(0) \geq 0$ and $\lim _{u \rightarrow g_{\max }} k_{y}(u)=+\infty$

- $g^{-1}(u)$ is monotone decreasing with respect to $u$ and $\lim _{u \rightarrow 0} g^{-1}(u)=+\infty$ and for $u=g_{\text {max }}$, $g^{-1}\left(g_{\max }\right)=0$,

then the two curves have a unique intersection point $u^{*}$ (see Figure 3 ) which present the unique fixed point of (19).

Global Attractivity. Denote by $T^{2}$ the composite function

$$
T^{2}(u)=(T \circ T)(u),
$$

where $T(u)=\left(g \circ k_{y}\right)(u)$. The following proposition gives the necessary and sufficient condition for the global attractivity of the unique equilibrium of (19).

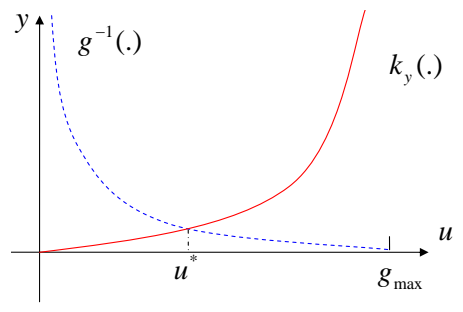

Figure 3: Graphical proof of the existence and unicity of the fixed point $u^{*}$ for the discrete system (19).

Proposition 4. If $u^{*}$ is also the unique fixed point of $T^{2}(u)$ on $\left(0, g_{\max }\right)$, That is

$$
\forall u \in\left(0, g_{\max }\right), T^{2}(u)=u \Leftrightarrow u=u^{*},
$$

then (19) converges to its unique fixed point.

Proof: see (Enciso and Sontag, 2006).

In practice, we can check condition (20) by graphical test $\left(\mathcal{H}_{3}\right)$. Indeed, if the graph of $T(u)$ and that of $T^{-1}(u)$ have a unique intersection point $u^{*}$ over $\left(0, g_{\text {max }}\right)$, then the composite function $T^{2}(u)$ has unique fixed point $u^{*}$. This completes the proof.

\subsection{Reversible Structure}

Now, consider the reversible metabolic pathways (1) and we prove that Proposition 1 is a consequence of Theorem 1.

Checking Assumption $\mathcal{H}_{5}$ : First, note that the enzyme $E_{1}$ is bounded (see proof given in subsection 4.1). Now, to analyze the boundedness of all the metabolite concentrations of (1), we proceed by step and we show that if any metabolite concentration $x_{i}$ is bounded then the metabolite concentration $x_{i-1}$ is also bounded. We start by $x_{2}$, and we consider the first differential equation of (1),

$$
\begin{aligned}
\dot{x}_{2} & =E_{1} f_{1}\left(\bar{x}_{1}, x_{2}, x_{n}\right)-E_{2} f_{2}\left(x_{2}, x_{3}\right) \\
& \leq \bar{E}_{1} f_{1}\left(\bar{x}_{1}, x_{2}, 0\right)-E_{2} f_{2}\left(x_{2}, x_{3}\right) .
\end{aligned}
$$

We assume that $x_{3}$ is bounded $\left(\forall t>0, x_{3}(t) \leq \bar{x}_{3}\right)$, then by definition there exists $x_{2}^{\star}$ such that:

$$
f_{1}\left(\bar{x}_{1}, x_{2}^{\star}, 0\right)=0 \text { and } f_{2}\left(x_{2}^{\star}, \bar{x}_{3}\right) \geq 0,
$$

and thus at $x_{2}^{\star}$ we obtain $\dot{x}_{2} \leq 0$. Hence the threshold $x_{2}^{\star}$ is repulsive, and so we have proved that the boundedness of $x_{3}$ implies the boundedness of $x_{2}$.

Now, for any metabolite concentration $x_{i}, i \in$ $\{3, \ldots, n-1\}$ we have $x_{i-1}$ bounded with bound $\bar{x}_{i-1}$, and we assume that $x_{i+1}$ is bounded with bound $\bar{x}_{i+1}$. Then the dynamics of $x_{i}$ is bounded by:

$$
\begin{aligned}
\dot{x}_{i} & =E_{i-1} f_{i-1}\left(x_{i-1}, x_{i}\right)-E_{i} f_{i}\left(x_{i}, x_{i+1}\right) \\
& \leq E_{i-1} f_{i-1}\left(\bar{x}_{i-1}, x_{i}\right)-E_{i} f_{i}\left(x_{i}, \bar{x}_{i+1}\right),
\end{aligned}
$$


and by definition we have

$$
\exists x_{i}^{\star} \mid f_{i-1}\left(\bar{x}_{i-1}, x_{i}^{\star}\right) \leq 0 \text { and } f_{i}\left(x_{i}^{\star}, \bar{x}_{i+1}\right) \geq 0 .
$$

Hence for $x_{i}^{\star}$ we obtain $\dot{x}_{i} \leq 0$, and so the threshold $x_{i}^{\star}$ is repulsive. Thus, we have proved that $\forall i \in$ $\{2, \ldots, n-1\}$ the boundedness of $x_{i+1}$ implies the boundedness of $x_{i}$. Lastly, consider the dynamics of the concentration of the end product $x_{n}$,

$$
\begin{aligned}
\dot{x}_{n} & =E_{n-1} f_{n-1}\left(x_{n-1}, x_{n}\right)-E_{n} f_{n}\left(x_{n}\right) \\
& \leq E_{n-1} M_{n-1}-E_{n} f_{n}\left(x_{n}\right) .
\end{aligned}
$$

Since $f_{n}($.$) is positive increasing and bounded with$ respect to $x_{n}$, it is clear that if $E_{n-1} M_{n-1} \leq E_{n} M_{n}$ we obtain

$$
\exists \bar{x}_{n} \mid \forall x_{n} \geq \bar{x}_{n} \Rightarrow \dot{x}_{n} \leq 0
$$

independently of the values of $x_{n-1}$. Consequently, if $\mathcal{H}_{2}$ is true, then all the state trajectories generated by (1) are bounded and so assumption $\mathcal{H}_{5}$ is verified.

Checking assumption $\mathcal{H}_{6}$ : As in the case of the irreversible metabolic pathways, structure (1) is not monotone. Nevertheless, we can decompose it into an open loop cooperative controlled system (21), which has tridiagonal Jacobian matrix $D F(x)$ with nonnegative off-diagonal entries, closed by a negative feedback (22).

- Open loop (cooperative system)

$$
\left\{\begin{array}{lll}
\dot{x}_{2} & = & E_{1} f_{1}\left(\bar{x}_{1}, x_{2}, g^{-1}(u)\right)-E_{2} f_{2}\left(x_{2}, x_{3}\right) \\
\dot{x}_{3} & = & E_{2} f_{2}\left(x_{2}, x_{3}\right)-E_{3} f_{3}\left(x_{3}, x_{4}\right) \\
\vdots & \vdots & \vdots \\
\dot{x}_{n} & = & E_{n-1} f_{n-1}\left(x_{n-1}, x_{n}\right)-E_{n} f_{n}\left(x_{n}\right) \\
\dot{E}_{1} & = & u-\mu E_{1} \\
y & = & x_{n}
\end{array}\right.
$$

- Negative feedback

$$
u=g(y)
$$

where $g^{-1}($.$) and g($.$) are the same as in the irre-$ versible case and also $u \in\left(0, g_{\max }\right)$. Hence, assumption $\mathcal{H}_{6}$ is intrinsically satisfied.

Checking assumption $\mathcal{H}_{7}$ : In the reversible context, build the static input-state characteristic is not explicit as in the irreversible case. However, to establish this characteristic we use the monotonicity property of all reaction rates $f_{i}(.,),. i \in\{1, \ldots, n\}$. First, we show that at steady state there exists a binary relation between each metabolite concentration $x_{i}, i \in\{3, \ldots, n\}$ and $x_{2}$. Second, we show that the metabolite concentration $x_{2}$ is an increasing function of the constant input $u$.
- Consider the dynamics corresponding to the last pool $X_{n}$. Since: $(i)$ the function $f_{n}\left(x_{n}\right)$ is monotone increasing in $x_{n}$ with $f_{n}(0)=0$, (ii) the function $f_{n-1}\left(x_{n-1}, x_{n}\right)$ is decreasing in $x_{n}$ and (iii) for any $x_{n-1}$ there exists $x_{n}^{*}$ such that $f_{n-1}\left(x_{n-1}, x_{n}^{*}\right)=$ 0 ,

$$
\forall x_{n-1}, \exists x_{n} \mid E_{n-1} f_{n-1}\left(x_{n-1}, x_{n}\right)=E_{n} f_{n}\left(x_{n}\right) .
$$

In other words, we can say that there exists a monotone increasing function $H_{n}($.$) with respect$ to $x_{n-1}$ such that:

$$
x_{n}=H_{n}\left(x_{n-1}\right) .
$$

- According to the previous stage, we can write

$$
f_{n}\left(x_{n}\right)=f_{n}\left(H_{n}\left(x_{n-1}\right)\right) .
$$

Thus, since $H_{n}($.$) is monotone increasing in x_{n-1}$, $f_{n}($.$) is also monotone increasing in x_{n-1}$. Now, consider the dynamics of the pool $X_{n-1}$. By definition $f_{n-2}\left(x_{n-2}, x_{n-1}\right)$ is decreasing in $x_{n-1}$ and for any $x_{n-2}$ there exists $x_{n-1}^{*}$ such that $f_{n-2}\left(x_{n-2}, x_{n-1}^{*}\right)=0$. Hence, we deduce: $\forall x_{n-2}$,

$$
\exists x_{n-1} \mid E_{n-2} f_{n-2}\left(x_{n-2}, x_{n-1}\right)=E_{n} f_{n}\left(H_{n}\left(x_{n-1}\right)\right) \text {. }
$$

Therefore, there exists a monotone increasing function $H_{n-1}($.$) with respect to x_{n-2}$ such that:

$$
x_{n-1}=H_{n-1}\left(x_{n-2}\right) \text { and } x_{n}=H_{n}\left(H_{n-1}\left(x_{n-2}\right)\right) \text {. }
$$

- Then we repeat this reasoning to obtain at steady state the following relations between $x_{2}$ and all the metabolic concentrations $x_{i}, i \in\{3, \ldots, n\}$ :

$$
\begin{aligned}
& x_{3}=H_{3}\left(x_{2}\right) \\
& x_{4}=H_{4}\left(H_{3}\left(x_{2}\right)\right) \\
& \vdots \\
& x_{n}=H_{n}\left(H_{n-1}\left(\ldots H_{3}\left(x_{2}\right)\right)\right)
\end{aligned}
$$

where all $H_{i}$ are increasing functions.

- Lastly, the enzyme's dynamics vanished while $E_{1}=\frac{u}{\mu}$. Thus, it is possible to build at the steady state a monotone relationship between the concentration of the pool $X_{2}$ and the input $u$. Indeed, as we have shown previously, $(i)$ the monotone decreasing property of the function $\frac{u}{\mu} f_{1}\left(\bar{x}_{1}, x_{2}, g^{-1}(u)\right)$ in $x_{2}$, (ii) the monotone increasing property of the function $f_{n}\left(H_{n}\left(H_{n-1}\left(\ldots H_{3}\left(x_{2}\right)\right)\right)\right.$ in $x_{2}$, and (iii) the existence of $x_{2}^{*}$ such that $f_{1}\left(\bar{x}_{1}, x_{2}^{*}, g^{-1}(u)\right)=0$ allow to state: $\forall u, \exists x_{2}$ such that,

$$
\frac{u}{\mu} f_{1}\left(\bar{x}_{1}, x_{2}, g^{-1}(u)\right)=f_{n}\left(H_{n}\left(H_{n-1}\left(\ldots H_{3}\left(x_{2}\right)\right)\right)\right.
$$

Then, at the steady state there exists a monotone increasing function $H_{2}($.$) with respect to u$ such that:

$$
x_{2}=H_{2}(u) \text {. }
$$


Hence, the static input-state characteristic of the system (21) is given by:

$$
\mathbf{k}_{x}^{T}(u)=\left[H_{2}(u), H_{3}\left(H_{2}(u)\right), \ldots, H_{n}\left(H_{n-1}\left(\ldots H_{2}(u)\right)\right), \frac{u}{\mu}\right]
$$

and its input-output characteristic is obtained by the composition law between (27) and the output equation of (21),

$$
k_{y}(u)=H_{n}\left(H_{n-1}\left(\ldots H_{2}(u)\right)\right) .
$$

Now, we must prove that for each constant input $u$ the vector $\left[\mathbf{x}^{* T}, \frac{u}{\mu}\right]=\mathbf{k}_{x}^{T}(u)$ is the globally asymptotically stable equilibrium point for the open loop system (21). To do so, we use the same analysis as in the irreversible case. First, we separate the two dynamics (enzymatic reaction, genetic regulation) and we deduce that for each constant input $u$ all the solutions generated by the dynamics of the genetic regulation $\left(\dot{E}_{1}\right)$ converge to $\frac{u}{\mu}$. Second, hypothesis $\mathcal{H}_{1}$ claims the existence of Tridiagonal Hurwitz matrix $\mathbf{Q}$ with nonnegative off-diagonal entries such that for all $\mathbf{x}$ the Jacobian matrix $D F(\mathbf{x})$ of the dynamics of the enzymatic reactions $\left(\dot{x}_{2}, \ldots, \dot{x}_{n}\right)$ is bounded by, $D F(\mathbf{x}) \leq \mathbf{Q}$. Then there exists a diagonal matrix $\mathbf{N}=\operatorname{diag}\left(n_{1}, \ldots, n_{n}\right)$ with $n_{i}>0$ and a real number $\varepsilon>0$ such that $\forall \mathbf{x}$

$$
\begin{aligned}
\mathbf{N} D F(\mathbf{x})+D F^{T}(\mathbf{x}) \mathbf{N} & \leq \mathbf{N Q}+\mathbf{Q}^{T} \mathbf{N} \\
& \leq-\varepsilon \mathbf{I}_{n-1}
\end{aligned}
$$

because $-\mathbf{Q}$ is a M-Matrix (Berman and Plemmons, 1994). Thus, the dynamics of the enzymatic reactions admits as Lyapunov function the quadratic form

$$
V(\mathbf{z})=\mathbf{z}^{T} \mathbf{N z},
$$

where $\mathbf{z}=\mathbf{x}-\mathbf{x}^{*}$. See previous demonstration of (18). Therefore, under assumption $\mathcal{H}_{1}$, relation (27) gives the globally asymptotically stable steady state of the open loop system (21) for each constant input $u$. This verifies assumption $\mathcal{H}_{7}$.

Finally, as we have shown in the context of irreversible metabolic pathways (here $\mathbf{k}_{x}(),. k_{y}($.$) and$ $g^{-1}($.) have the same properties with respect to $u$ as in the irreversible context), we can check the global convergence of the following scalar discrete time dynamical system

$$
u_{j+1}=g\left(H_{n}\left(H_{n-1}\left(\ldots H_{2}\left(u_{j}\right)\right)\right),\right.
$$

to its unique fixed point $u^{*} \in\left(0, g_{\max }\right)$ by the same graphical test stated in assumption $\left(\mathcal{H}_{3}\right)$. This completes the proof that Proposition 1 is a consequence of Theorem 1.

\section{CONCLUSIONS}

We have used in this paper the negative feedback theorem of monotone control SISO systems theory, to give technical propositions which prove global attractivity of linear metabolic pathways. For future works, we will consider the stability analysis for dynamical systems through monotone control MIMO systems. That will allow us to tackle the stability issue for complex bacterial metabolic networks.

\section{REFERENCES}

Angeli, D. and Sontag, E. D. (2003). Monotone control systems. IEEE transactions on automatic control, 48:1684-1698.

Angeli, D. and Sontag, E. D. (2004). Multi-stability in monotone input/output systems. Systems and Control Letters, 51:185-202

Angeli, D. and Sontag, E. D. (2008). Oscillations in i/o monotone systems under negative feedback. IEEE transactions on automatic control, 55:166-176.

Arcak, M. and Sontag, E. D. (2006). Diagonal stability of a class of cyclic systems and its connection with the secant criterion. Automatica, 42:1531-1537.

Berman, A. and Plemmons, R. (1994). Nonnegative Matrices in the Mathematical Sciences. Society for Industrial and applied Mathematics, Philadelphia.

Enciso, G. A. and Sontag, E. D. (2006). Global attractivity, i/o monotone small-gain theorems, and biological delay systems. Discrete and Continuous Dynamical Systems, 14:549-578.

Goelzer, A., Bekkal-Brikci, F., Martin-Verstraete, I., Noirot, P., Bessiéres, P., Aymerich, S., and Fromion, V. (2008). Reconstruction and analysis of the genetic and metabolic regulatory networks of the central metabolism of bacillus subtilis. BMC Systems Biology, doi:10.1186/1752-0509-2-20.

Gollnick, P., Babitzke, P., Antson, A., and CA, C. (2005). Complexity in regulation of tryptophan biosynthesis in bacillus subtilis. Annual review of genetics, 39:4768.

Grundy, F., Lehman, S., and Henkin, T. (2003). The 1 box regulon: lysine sensing by leader rnas of bacterial lysine biosynthesis genes. PNAS, 100(21):1205712062.

Sanchez, L. (2009). Global asymptotic stability of the goodwin system with repression. Nonlinear analysis : real world applications, 10(4):2151-2156.

Smith, H. L. (1995). Monotone dynamical systems: An Introduction to the theory of competitive and cooperative systems. Mathematical Surveys and Monographs Vol. 41, AMS, Providence, RI.

Tyson, J. and Othmer, H. (1978). The dynamics of feedback control circuits in biological pathways. In R. Rosen, and F.M. Snell (Eds.), Progress in theoretical biology (Vol. 5), pages 1-62, New York: Academic Press.

Wang, L., de Leenheer, P., and Sontag, E. (2008). Global stability for monotone tridiagonal systems with negative feedback. In Proc. IEEE Conf. Decision and Control CDC, pages 4091-4096, Cancun,Mexico. 